\title{
The progress and current status of Tokamak: a systematic review
}

\author{
Yuxuan Song*1 \\ ${ }^{1}$ Pioneer academy school, Changzhou, Jiangsu, 213000, China
}

\begin{abstract}
Nuclear fusion energy is an ideal energy source for the future due to the clean and efficient features, first proposed by Russian scientists in the 1950s. With the successful construction and operation of a batch of tokamak devices globally, a series of major achievements have been realized in magnetic confinement fusion (MF). On this basis, the recent progress and current status of tokamak devices are systematically reviewed from academic literatures and official websites. To better demonstrate, the principle of MF and the history of a tokamak are introduced firstly. Subsequently, developments of four typical state-of-art tokamak facilities (JT60, FTFR, JET, EAST) are discussed detailly. These results offer a guideline for tokamak device construction and MCF realization.
\end{abstract}

\section{Introduction}

Nuclear fusion is a reaction that combines two or more atom nuclei to form a heavier atom nuclear and other matters (e.g., protons, neutrons, and leptons). After the reaction, there will be a difference between the reactants and products by either releasing or absorbing energy because of the atomic binding energy. Fusion is the process that powers active or main sequence stars and other high-magnitude stars. The deuterium-tritium (D-T) reaction is the most appealing one for confinement fusion power plants among all the fusion processes due to the largest cross-section. Besides, the deuterium source is plentiful on the earth, while the tritium can be obtained from lithium. Fusion power plants will not produce greenhouse gases, i.e., they can contribute a large part of the future energy structure without causing global warming. The realization of fusion energy is one of the major challenges facing mankind, which will bring huge social benefits of success.

Contemporarily, the fusion research communities try their best to solve the remaining challenges, which have made lots of progress. Thereinto, Tokamak is one of the most attractive ones that close to the goal [1]. The word tokamak is a transliteration of the Russian word токамак, which is actually an abbreviation. Its name Tokamak comes from ring(toroidal), vacuum chamber (kamera), magnetism (magnit), coil (kotushka). Soviet Union scientists in the 1950s proposed the tokamak. In 1958, the first real tokamak device was built $[2,3]$. The rest of the paper is organized as follows. Primarily, the principle of magnetic confinement fusion (MCF) and the history of Tokamak are introduced in Sec. 2. Then, the progress of four different devices (JT60, FTFR, JET, EAST) is demonstrated in Sec. 3. Finally, a summary is presented in Sec. 4.

\section{The principle of operation and history of Tokamak}

To achieve a D-T fusion reaction successfully, one must meet the Lawson criterion, where the products of density, temperature, and confinement time ought to reach $3 \times 10^{21}$ $\mathrm{keV} \cdot \mathrm{s} \cdot \mathrm{m}^{-3}$. On this basis, it requires heating the nuclear fuel to over 100 million ${ }^{\circ} \mathrm{C}$ to form plasma. However, it is almost impossible for any material on the earth to endure such a high temperature. As a result, magnetic fields are designed to realize the confinement of plasma without direct contact, known as MCF. The tokamak is one of the renewable configurations of MCF devices. Typically, a tokamak is a device that uses a powerful magnetic field to confine hot plasma in the shape of a torus. The tokamak is one of several types of magnetic confinement devices developed to produce controlled thermonuclear fusion power. In 2016, it was the leading candidate for a practical fusion reactor. As the most advanced and mature fusion device so far globally, Tokamak uses a magnetic field to confine the charged particles of energy and plasma in an annular cavity. This structure is called a magnetic trap. The magnetic field has two orthogonal components: one is generated by a D-shaped coil system surrounding the current the plasma, and the other is generated by the current induced in the plasma. The generated magnetic field lines spiral around a set of nested annular magnetic flux surfaces, thereby providing an effective plasma confinement system that can be heated to conditions close to the conditions required for fusion energy. In 1997, experiments were carried out in the European Union Circle (JET) tokamak, using deuterium and tri to generate $16 \mathrm{MW}$ of fusion energy while requiring $25 \mathrm{MW}$ of

*yuxuan.song@pioneeracademy.org 
plasma heating. Although the energy which the experience used is much bigger than the energy tokamak produced, it is still a great step in the controlled fusion industry.

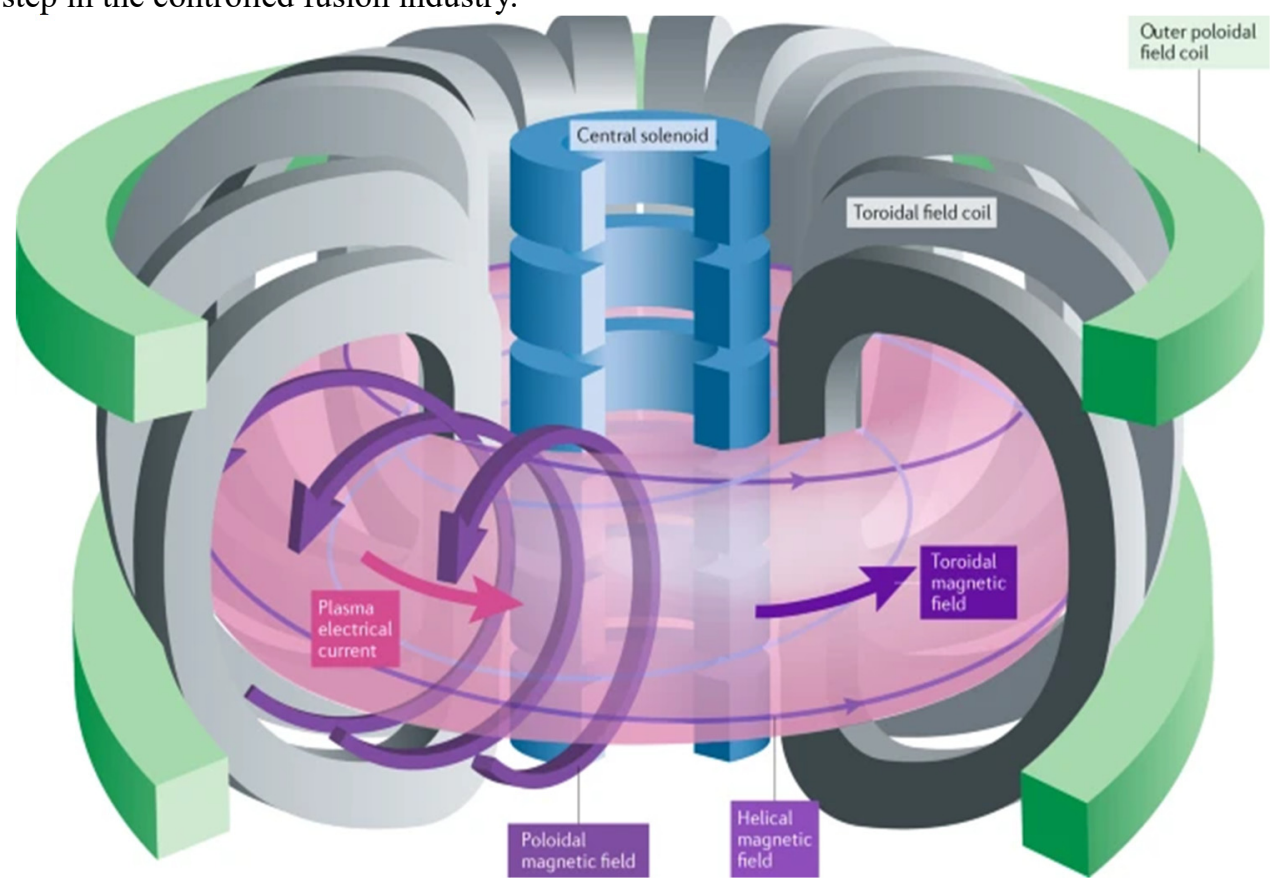

Figure 1. A sketch of Tokamak [2]

As shown in Fig. 1, a typical tokamak configuration is made up of a central solenoid, toroidal field coil, and outer poloidal field coil. It uses a current-carrying coil to generate part of the required magnetic field. Electric current is induced in the plasma to generate a further magnetic field, thereby generating spiral magnetic field lines. Leading the way is that the charged particles of the plasma follow these magnetic lines of force, thus creating an effective plasma confinement system. A snapshot of a real device (EUROfusion T-15MD) is illustrated in Fig. 2.

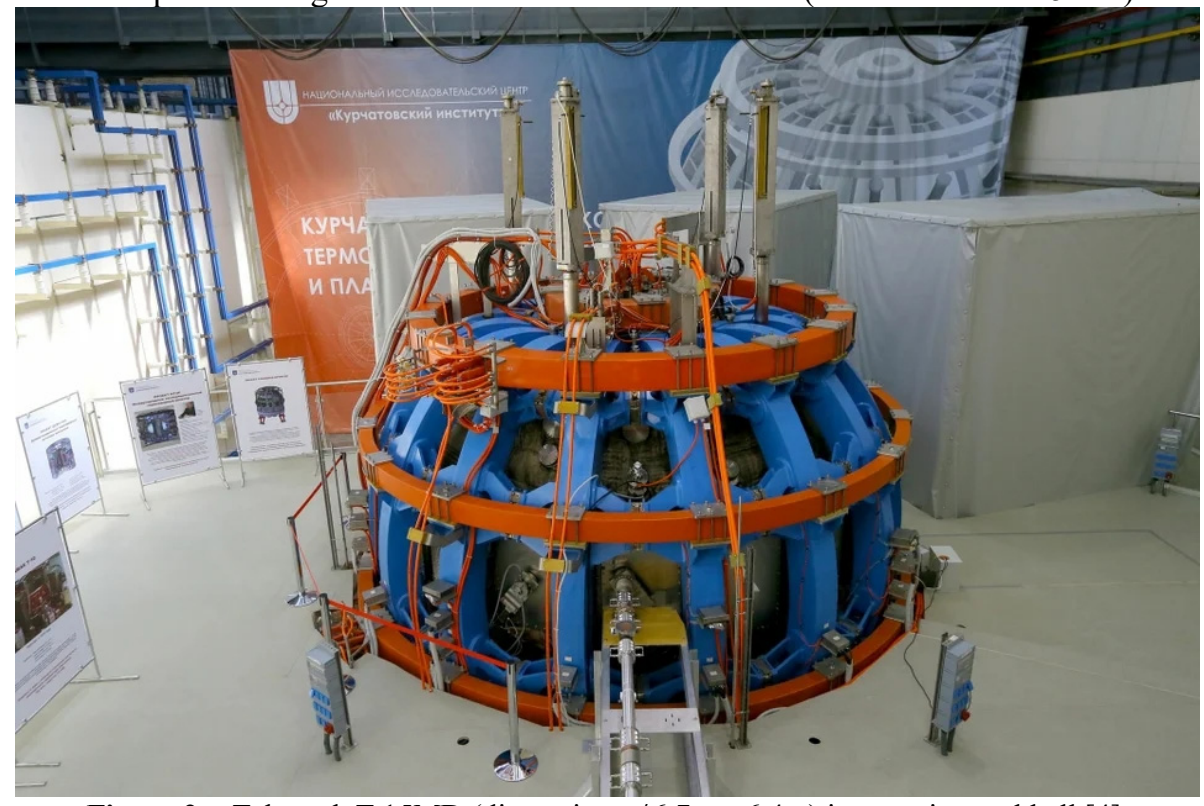

Figure 2. Tokamak T-15MD (dimensions $\emptyset 6.7 \mathrm{~m} \times 6.4 \mathrm{~m}$ ) in experimental hall [4].

According to the Maxwell equation in vacuum condition, the magnetic induction intensity gradient $\nabla \mathrm{B}$ and the bending magnetic field will inject kinetic energy $\mathrm{W} \perp$ perpendicular to the direction of motion and kinetic energy W// parallel to the direction of motion, causing the particles to gradually deviate from the direction of the magnetic line of induction. This phenomenon is called "particle drift". Because ions and electrons drift in opposite directions, the ions in the nuclear fuel will gradually gather to the upper part while the electrons will gather to the lower part. Consequently, the separation of positive and negative charges will produce a vertical downward "ghost" electric field. It will work with the toroidal magnetic field to produce a velocity component directed outside the solenoid to the particles. Hundreds of millions of degrees of nuclear fuel will be thrown out. Thus, the second set of energized coils must be sheathed outside the toroidal magnetic field, which can generate a vertical magnetic field. On this basis, the two magnetic fields synthesize a spiral magnetic field around the major 
axis. In this case, the electrons collected in the lower part can be transferred to the upper part and mixed with the ions along the spiral magnetic field, and the ions collected in the upper part can also be transferred to the lower part. The particles are evenly stirred, and the "ghost" electric field disappears without a trace.

After successfully restraining the nuclear fuel, it is necessary to consider the ignition and heating of the nuclear fuel. This requires the addition of the third set of energized coils-central solenoid coils in the center of the device. It can provide a rapidly changing magnetic flux through the nuclear fuel ring, induce a ring electromotive force in the nuclear fuel, break down to form a plasma, and generate an induced current inside it. The ohmic heat generated by the electric current makes the nuclear fuel continue to warm up. With various auxiliary heating methods such as radiofrequency electromagnetic waves and neutral particle beam injection, the nuclear fuel will eventually reach the fusion condition of 100 million degrees and ignite the artificial sun.

\section{Progress of Tokamak devices worldwide}

This section mainly reviews four types of state-of-art tokamak devices around the world, including JT-60, TFTR, JET, and EAST.

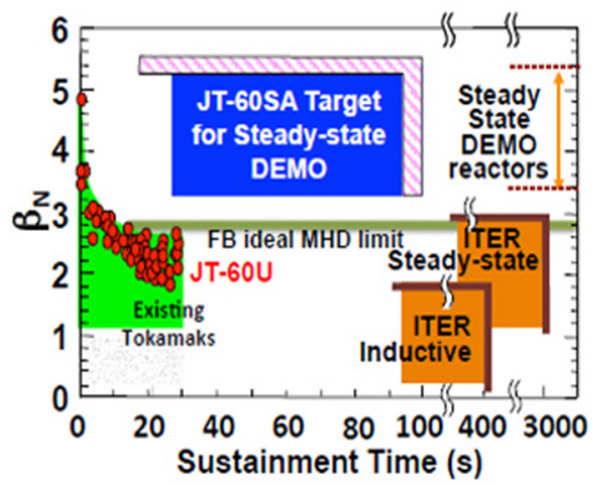

Figure 3. Its performance range, compared to other experiments worldwide, as well as with expected ITER and DEMO operation, is shown above $(\mathrm{nD}(0)=$ plasma central ion density, $\tau \mathrm{E}=$ energy confinement time, $\mathrm{Ti}(0)=$ plasma central ion temperature, $\beta \mathrm{N}=$ normalised beta) [5]

\subsection{TFTR}

The Tokamak Fusion Test Reactor (TFTR) was an experimental tokamak built at Princeton Plasma Physics Laboratory (PPPL) circa 1980 and entering service in 1982. TFTR was designed with the explicit goal of reaching scientific breakeven. The point where the heat is released from the fusion reactions in the plasma is equal or greater than the heating being supplied to the plasma by external devices to warm it up. The physical goal of TFTR is to explore and understand fusion reactor deuteriumtritium (D-T) Plasma behavior characteristics of the plasma core. The layout of TFTR has depicted in Fig. 4. The body performance is close to the predicted D-T fusion reactor plasma performance. This should be contributed to

\subsection{JT-60SA}

JT-60SA is a joint international fusion experiment being built and operated by Japan and Europe in Naka, Japan, using the infrastructure of the previous JT-60 Upgrade experiment alongside new hardware. SA stands for "super, advanced", since the experiment will have superconducting coils and study advanced modes of plasma operation. JT-60SA has been designed to support the operation of ITER by following a complementary research and development programme and to investigate how best to optimise the operation of fusion power plants that are built after ITER. The performance range of JT60SA and a comparison of other facilities are shown in Fig. 3. When fully operational, JT-60SA will be able to sustain break-even-equivalent high-temperature deuterium plasmas for typically $100 \mathrm{~s}$, longer than the timescales characterising key plasma processes such as current diffusion and particle recycling. The maximum plasma current its 5.5 MA. The device can also pursue fully noninductive steady-state operation with values of the plasma pressure exceeding plasma stability limits without wallstabilisation.

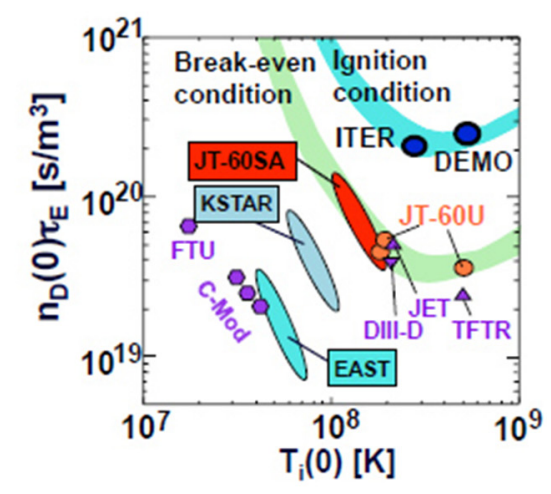

studying the plasma core related to the D-T fusion reactor, Ion transport, magnetic fluid (MHD) instability, and alpha-particle physics. The main research results of TFTR include: obtained the relevant fusion reactor, Large-scale D-T plasma confinement, heating and alpha-particle physics, specific information, and tritium processing and $\mathrm{D}-\mathrm{T}$ in the experimental environment.

As a matter of fact, the fusion power density is similar for the devices and ITER, where the peak fusion power of $\mathrm{D}-\mathrm{T}$ plasma reaches $10.7 \mathrm{MW}$, and the central fusion power density is $2.8 \mathrm{MW} \cdot \mathrm{m}^{-3}$ while $1500 \mathrm{MW}$ fusion power and $1.7 \mathrm{MW} \cdot \mathrm{m}^{-3}$ for ITER. With three years of TFTR's operation in D-T, D-T plasma-related research has achieved significant development. 


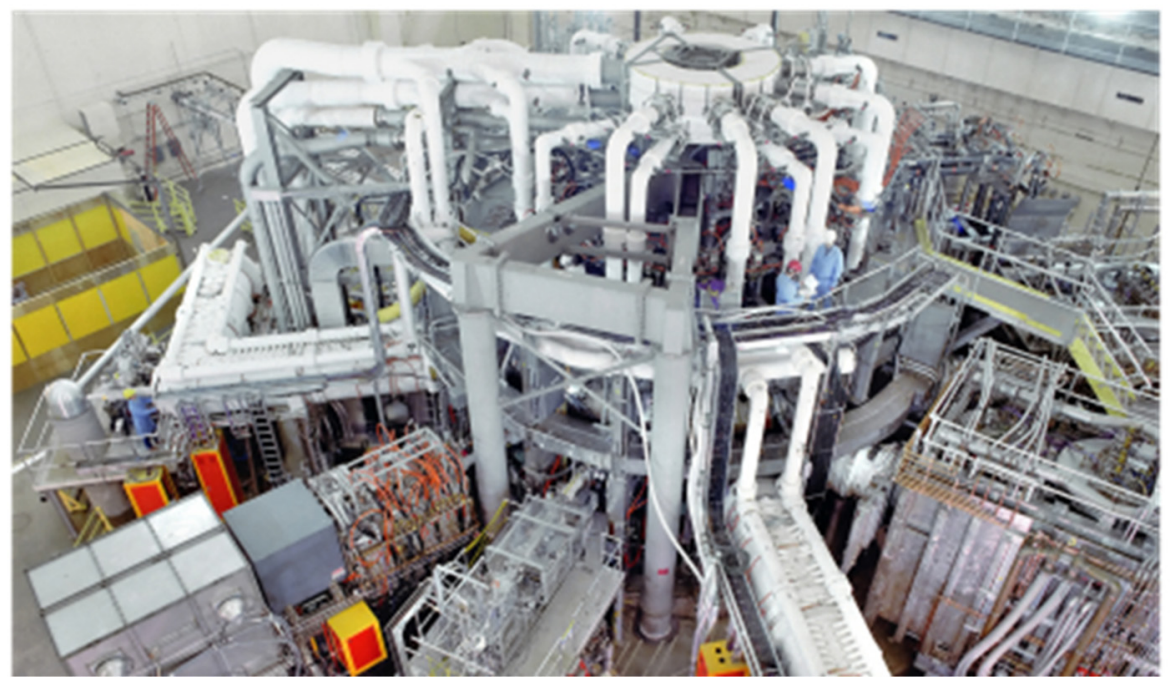

Figure 4. Aerial view of the Tokamak Fusion Test Reactor [6].

\subsection{JET}

JET, operated at the Culham Centre for Fusion Energy (CCFE), UK, is the central research facility of the European Fusion Programme. It is one of the largest and most successful fusion experiments in the world. JET is collectively used under EUROfusion's management by more than 40 European laboratories. Over 350 scientists and engineers from all over Europe contribute to the JET programme [7]. Since 1983, JET was explicitly designed to study plasma behaviour in conditions and dimensions approaching those required in a fusion reactor. So far, its primary task is to prepare for the construction and operation of ITER, acting as a testbed for ITER technologies and plasma operating scenarios.

The core of JET is a vacuum vessel, which limits the fusion plasma through a strong magnetic field and plasma current (up to 4 Tesla and five mega amperes). In the current configuration, the plasma ring's major radius and minor radius are 3 meters and 0.9 meters, respectively, and the total plasma volume is 90 cubic meters. The flow divider at the bottom of the vacuum vessel allows heat and gas to escape remotely. Since 2011, the first wall of the vacuum vessel has been made of beryllium and tungsten, similar to ITER's material selection. That is also the reason why JET is called The Little ITER [8].

\subsection{EAST}

EAST (HT-7U Superconducting Tokamak), a non-circular advanced steady-state plasma experimental device, was rectified by the Leading Team of Science and Technology, the State Council on June 3, 1997, as a National MegaProject of Science Research. Its scientific mission is to realize stable operation, carry out experiments on heating and confinement improvement under the tokamak advanced operation, and finally realize the tokamak advanced operational mode. It has a long pulse (60-1000s) capability, a flexible PF system, and auxiliary heating and current drive systems. It will accommodate divertor heat loads that make it an attractive test facility for the development of advanced tokamak operating modes. HT-
$7 \mathrm{U}$ consists of nine sub-systems: superconducting tokamak device, cryogenic and refrigerator system, power supply system, control and data acquisition system, lower hybrid current drive (LHCD) system, ion cyclotron resonance heating (ICRH) system, vacuum pumping and gas puffing system, diagnostic system, and water-cooling system [9]. Recently, it realizes the new records of hightemperature plasma confinement, where the confinement time reaches the 20 s for 160 million ${ }^{\circ} \mathrm{C}$ and $101 \mathrm{~s}$ for 120 million ${ }^{\circ} \mathrm{C}$, respectively [10].

\section{Conclusion}

In summary, the progress and current status of tokamak devices are evaluated in this review. The history and basic principles of MCF and tokamak are introduced primarily to better introduce the challenges and gaps for the devices. Then, four well-known tokamak devices are discussed specifically, including JT60, FTFR, JET, EAST. In detail, the configurations, operation parameters, and future goals of these devices are demonstrated. With their ambitious scheme, the realization of confinement fusion will come true in quantitative time. These results shed light for future state-of-art MCF device operation and construction.

\section{References}

1. Pironti, Alfredo, and Michael Walker. "Control of tokamak plasmas: introduction to a special section." IEEE Control Systems Magazine 25.5 (2005): 24-29.

2. Smirnov, V. P. "Tokamak foundation in USSR/Russia 1950-1990." Nuclear fusion 50.1 (2009): 014003.

3. Fisch, Nathaniel J. "Confining a tokamak plasma with rf-driven currents." Physical Review Letters 41.13 (1978): 873.

4. https://www.reddit.com/r/europe/comments/ng0jum/ the_launch_of_the_t15md_tokamak_thermonuclear/

5. https://www.jt60sa.org/wp/

6. https://www.pppl.gov/news/2020/09/groundbreaking -tokamak-fusion-test-reactor-designated-nuclearhistoric-landmark 
7. https://www.euro-fusion.org/devices/jet/

8. Romanelli, Michele, et al. "JINTRAC: a system of codes for integrated simulation of Tokamak scenarios." Plasma and Fusion research 9 (2014): 3403023-3403023.

9. http://english.hf.cas.cn/ic/ip/east/

10. https://baijiahao.baidu.com/s?id=170106853454593 $3093 \& \mathrm{wfr}=$ spider $\&$ for $=\mathrm{pc}$ 